\title{
STANDARD PRIME IDEALS AND LYING OVER FOR FINITE EXTENSIONS OF NOETHERIAN ALGEBRAS
}

\author{
by GÜNTER KRAUSE $\dagger$
}

(Received 10 March, 1994)

Introduction. Let $k$ be a field, let $R$ be a noetherian $k$-algebra of finite Gelfand-Kirillov dimension $\mathrm{GK}(R)$, and let $M$ be a finitely generated right $R$-module. A standard prime factor series for $M$ is a finite sequence of submodules $0=N_{0} \subset N_{1} \subset \ldots \subset$ $N_{i-1} \subset N_{i} \subset \ldots \subset N_{n}=M$, such that for each $i$ the annihilator $P_{i}=r_{R}\left(N_{i} / N_{i-1}\right)$ is the unique associated prime of $N_{i} / N_{i-1}$ and $\operatorname{GK}\left(R / P_{i}\right) \leq \mathrm{GK}\left(R / P_{j}\right)$ whenever $i \leq j$. The set of prime ideals arising from such a series is an invariant of $M$, called the set of standard primes $\operatorname{St}(M)$ of $M$. The concept, inspired by the notion of a standard affiliated series introduced by Lenagan and Warfield in [7], has been developed in [5], where it was shown that $\operatorname{St}(M)$ coincides with the set of all those prime ideals that are minimal over the annihilator of a nonzero submodule of $M$.

In general, one cannot expect to have any bound on the lengths of the various standard prime factor series of $M$, the case when $M=R_{R}$ is a simple noetherian domain that is not a division algebra furnishes a counterexample. However, the situation changes when ${ }_{S} M_{R}$ is a bimodule, finitely generated on each side, where $R$ and $S$ are noetherian $k$-algebras of finite GK-dimension. In Section 1, it is shown that any standard prime factor series of $M_{R}$ whose terms are $S$-R-sub-bimodules $N_{i}$ does have bounded length, the least upper bound being called the (right) standard length of $M$. It is realized as the length of any unrefinable such standard series. Furthermore, the left standard length of $M$ equals the right standard length, and any unrefinable standard prime factor series of $M_{R}$ that consists of sub-bimodules is automatically a standard prime factor series of ${ }_{s} M$.

In Section 2, we briefly discuss the multiplicity of a standard prime $P$, that is the number of times $P$ occurs as the right annihilator of ${ }_{s}\left(N_{i} / N_{i-1}\right)_{R}$ for a given right standard prime factor series of ${ }_{s} M_{R}$. It is shown that the multiplicity of $P$ in any two such unrefinable series is the same.

The characterization of standard primes as prime ideals that are minimal over the annihilator of a submodule suggests their use in questions related to "lying over". For this, let $R$ be a noetherian subalgebra of the noetherian $k$-algebra $S$, such that $S_{R}$ is finitely generated and $\mathrm{GK}(R)$ is finite. A prime ideal $Q$ of $S$ is said to lie over the prime ideai $P$ of $R$ if $P$ is minimal over $Q \cap R$. In general, a prime $P$ of $R$ need not have LO, that is, there need not exist a prime ideal of $S$ that lies over $P$. Thus, one is faced with the problem of finding criteria that assure lying over for a given prime of $R$. In Section 3, it is shown that any standard prime $P$ of $R_{R}$ has LO and that if $Q$ lies over $P$, then $Q \in \operatorname{St}\left(S_{S}\right)$. This leads to the following criterion for lying over: $P \in \operatorname{spec}(R)$ has LO precisely when $P \in \mathrm{St}(S / I)_{R}$ for some ideal $I$ of $S$. Subsequently, at the end of Section $4, P$ is shown to have LO if and only if $P \in \operatorname{St}\left({ }_{R} X\right)$ for some finitely generated left $R$-submodule $X$ of a left $S$-module $M$.

In Section 4, we consider the more restrictive property of lying directly over, introduced by Goodearl and Letzter in [1]. A prime ideal $Q$ of $S$ is said to lie left directly

$\dagger$ The author gratefully acknowledges support from the Natural Sciences and Engineering Research Council of Canada.

Glasgow Math. J. 37 (1995) 311-326. 
over the prime ideal $P$ of $R$ if $P$ is the left annihilator of a left $R$-submodule of $S / Q$. Using standard primes, it is shown that $P$ has left directly lying over if and only if $S P \cap R=P$. Furthermore, at most a finite number of primes of $S$ can lie left directly over a given prime of $R$.

In general, it is not known whether only a finite number of primes of $S$ can lie over a given prime of $R$, although Letzter [10] has recently provided a positive answer for a class of rings that includes complex group algebras over polycyclic-by-finite groups, noetherian PI algebras, and enveloping algebras of finite dimensional complex solvable or semisimple Lie algebras. In Section 5, we present some partial results. Specifically, it is shown that a prime $P$ of $R$ has finite lying over whenever $\mathrm{GK}(R / P)$ is greater than or equal to $\mathrm{GK}\left(R / r_{R}(S / R)\right)$. This extends a result due to Small and Warfield [14], who assumed both $R$ and $S$ to be prime, both $S_{R}$ and ${ }_{R} S$ to be finitely generated, and $R_{R}$ to be essential in $S_{R}$. Finally, an extreme situation arises when $\mathrm{GK}(R / P)>\mathrm{GK}_{R}(S / R)$. In this case, precisely one prime ideal $Q$ of $S$ lies over $P$ and $P=Q \cap R$.

Definitions AND notations. All rings considered are (left and right) noetherian algebras over a field $k$, modules are unitary. The Gelfand-Kirillov dimension, GKdimension for short, of a right $R$-module $M$ is denoted by $\mathrm{GK}(M)$. If $M$ is an $S$ - $R$-bimodule, finitely generated on both sides, then $\mathrm{GK}\left({ }_{s} M\right)=\mathrm{GK}\left(M_{R}\right)$, and the subscript is deleted. Only when the bimodule is not finitely generated on one side a subscript is used on the appropriate side. For basic properties of GK-dimension we refer to [6]. Most of the terminology is standard and follows the book by Goodearl and Warfield [2].

$$
\begin{aligned}
r_{R}(X) & =r(X)=\text { right annihilator in } R \text { of a subset } X \text { of a right } R \text {-module. } \\
l_{R}(X) & =l(X)=\text { left annihilator in } R \text { of a subset } X \text { of a left } R \text {-module. } \\
\mathscr{C}(I) & =\text { set of elements of } R \text { that are regular modulo the ideal } I \text { of } R . \\
\operatorname{spec}(R) & =\text { set of prime ideals of the ring } R . \\
\text { annspec }(M) & =\left\{P \in \operatorname{spec}(R) \mid P=r(X), 0 \neq X \subseteq M_{R}\right\}
\end{aligned}
$$

A prime ideal $P$ of $R$ is associated with the right $R$-module $M$ if $P=r\left(N^{\prime}\right)$ for all submodules $N^{\prime} \neq 0$ of some nonzero submodule $N$ of $M$. The submodule $N$ is called a prime submodule of $M$.

$\operatorname{Ass}(M)=$ set of primes associated with the module $M$.

$\rho(M)=\rho(M)_{R}=$ reduced rank of the right $R$-module $M$.

$\operatorname{rank}(M)=$ uniform (Goldie) rank of the module $M$.

1. Standard length of a bimodule. Let $R$ be a noetherian $k$-algebra, and let $M$ be a finitely generated right $R$-module. A sequence of submodules $0=N_{0} \subset N_{1} \subset \ldots \subset N_{i-1} \subset$ $N_{i} \subset \ldots \subset N_{n-1} \subset N_{n}=M$ with $P_{i}=r\left(N_{i} / N_{i-1}\right)=\operatorname{Ass}\left(N_{i} / N_{i-1}\right)$ is called a prime factor series of $M$. It is called standard if $\mathrm{GK}\left(R / P_{i}\right) \leq \mathrm{GK}\left(R / P_{j}\right)$ whenever $i \leq j$. If $B \subset C \subset D$ are three consecutive terms of such a series such that $\mathrm{GK}(R / r(C / B))<\mathrm{GK}(R / r(D / C))$, then $C$ is called a jumping point of the series. A standard prime factor series has $s-1$ such jumping points where $s$ denotes the number of distinct real numbers in the set $\{\mathrm{GK}(R / P) \mid P \in \mathrm{St}(M)\}$. By Theorem 2.4 of [5], any two standard prime factor series have the same jumping points, they will be denoted by $J_{i}=J_{i}(M), 0 \leq i \leq s$, setting $J_{0}(M)=0$ and $J_{s}(M)=M$, for convenience. It is clear that for each $i, J_{i}(M) / J_{i-1}(M)$ is equidimensional in the following sense. 
Definition. A finitely generated right $R$-module $M$ over the noetherian $k$-algebra $R$ is equidimensional if $\mathrm{GK}(R / P)=\mathrm{GK}(R / Q)$ for any two standard primes $P$ and $Q$ of $M$.

LEMMA 1.1. Let $R$ be a noetherian $k$-algebra, and let $M$ be a finitely generated right $R$-module with $\mathrm{GK}(R / r(M))<\infty$. If $M$ is equidimensional, then

$$
\operatorname{St}(M)=\{P \in \operatorname{spec}(R) \mid P \text { minimal over } r(M)\} \text {. }
$$

Proof. By [5, Theorem 3.3], any prime that is minimal over $r(M)$ is a standard prime of $M$. Let $P \in \mathrm{St}(M)$, and assume that $P \ni Q \supseteq r(M)$ with $Q$ minimal over $r(M)$. Since $Q$ is standard, and since $\mathrm{GK}(R / P)<\mathrm{GK}(R / Q)$, this contradicts equidimensionality.

Definition. Let $R$ and $S$ be noetherian $k$-algebras, and let ${ }_{s} M_{R}$ be a bimodule that is finitely generated on either side. A sequence of sub-bimodules

$$
0=B_{0} \subset B_{1} \subset \ldots \subset B_{i-1} \subset B_{i} \subset \ldots \subset B_{n}=M
$$

that is a standard prime factor series of $M_{R}\left({ }_{s} M\right)$ is called a right (left) standard prime bi-factor series of $M$.

Right (or left) standard prime bi-factor series of a bimodule exist by [5, Proposition 2.3], any right (left) standard affiliated series of $M$ will do.

LEMMA 1.2. Let $R$ and $S$ be noetherian $k$-algebras, let ${ }_{S} M_{R}$ be a bimodule, finitely generated on both sides, with $\mathrm{GK}(M)=\mathrm{GK}(R / r(M))<\infty$, and assume that $M_{R}$ is equidimensional. Then the length of a right standard prime bi-factor series of $M$ is bounded by $\rho(M)_{R / r(M)}$, the reduced rank of $M$ as a right $R / r(M)$-module.

Proof. Let $0=B_{0} \subset B_{1} \subset \ldots \subset B_{i-1} \subset B_{i} \subset \ldots \subset B_{n}=M$ be a right standard prime bi-factor series of $M$ with $P_{i}=r_{R}\left(B_{i} / B_{i-1}\right)=\operatorname{Ass}\left(B_{i} / B_{i-1}\right)_{R}$. By Lemma 1.1, each $P_{i}$ is minimal over $r_{R}(M)$. Note that each $B_{i} / B_{i-1}$, being finitely generated on the left, is $\mathscr{C}\left(P_{i}\right)$-torsionfree. Thus it follows as in the proof of [4, Theorem 5.1] (note that the proof does not require the $B_{i}$ 's to be annihilator submodules of $M$ ) that

$$
\rho(M)_{R / r(M)}=\sum_{i=1}^{n} \operatorname{rank}\left(B_{i} / B_{i-1}\right)_{R} \geq n .
$$

Proposition 1.3. Let $R$ and $S$ be noetherian $k$-algebras, let ${ }_{s} M_{R}$ be a bimodule, finitely generated on both sides, and assume that $\mathrm{GK}(M)<\infty$. Then the length of any right standard prime bi-factor series of $M$ is bounded by

$$
\sum_{i=1}^{s} \rho\left(J_{i} / J_{i-1}\right)_{R / r\left(J_{i} / J_{i-1}\right)}
$$

Proof. As such a series, like any standard prime factor series of $M_{R}$, passes through the jumping points $J_{i}$, and since each of the bimodules $J_{i} / J_{i-1}$ satisfies the hypotheses of Lemma 1.2, the claim follows.

In the situation under consideration, one always has that $\mathrm{GK}\left(S / l_{S}(M)\right)=\mathrm{GK}(M)=$ $\mathrm{GK}\left(R / r_{R}(M)\right)$ (see [6,5.3 and 5.4]), so one can compare the maximal length of a right standard prime bi-factor series of $M$ with that of such a series on the left. As will be shown below, these are the same. 
Definition. Let $R$ and $S$ be noetherian $k$-algebras, and let ${ }_{s} M_{R}$ be a bimodule, finitely generated on both sides, with $\mathrm{GK}(M)<\infty$. The right (left) standard length r.stL $(M)(1 . s t L(M))$ is the maximal length of a right (left) standard prime bi-factor series of $M$. Such a series is full if it is not refinable, that is, if no sub-bimodule can be inserted between any two consecutive terms of the series to obtain a standard prime bi-factor series of greater length.

Trivially, any right (left) standard prime bi-factor series of maximal length is full, and we proceed to show that any two full right standard prime bi-factor series have the same length r.stL $(M)$.

Lemma 1.4. Let $R$ and $S$ be noetherian $k$-algebras, let ${ }_{s} M_{R}$ be a bimodule that is finitely generated on both sides, let $\mathrm{GK}(M)<\infty$, and assume that $\operatorname{r.stL}(M)=1$. Then:

(i) $M / X$ is $\mathscr{C}(P)$-torsion for any nonzero sub-bimodule $X \subseteq M$, where $P=r_{R}(M)=$ $\operatorname{Ass}(M)_{R}$;

(ii) $\operatorname{r.stL}(N)=1$ for any nonzero sub-bimodule $N \subseteq M$.

Proof. (i) Assume this to be false, and let $X$ be maximal among nonzero subbimodules with $M / X$ not $\mathscr{C}(P)$-torsion. Clearly, $r(M / X)=P$. Now Ass $(M / X)_{R} \neq P$, for otherwise $0 \subset X \subset M$ would be a right standard prime bi-factor series of length 2 . Therefore, let $Y$ be a sub-bimodule, $Y \ni X, r(Y / X)=Q \neq P$. Since $r(Y / X) \supseteq r(M / X)=$ $P$, it follows that $Q \supsetneqq P$, so $Y / X$ is $\mathscr{C}(P)$-torsion. As $M / Y$ is $\mathscr{C}(P)$-torsion by the maximality of $X$, this leads to a contradiction.

(ii) Assume there exists a right standard prime bi-factor series of $N$ of length greater than 1, with, say, $0 \neq N^{\prime} \subset N$ at the top. As $\operatorname{St}(N) \subseteq \operatorname{St}(M)=\{P\}$ by [5, Corollary 3.5], $P=r\left(N / N^{\prime}\right)=\operatorname{Ass}\left(N / N^{\prime}\right)_{R}$. On the other hand, $N / N^{\prime} \subseteq M / N^{\prime}$ is $\mathscr{C}(P)$-torsion by (i). As $s\left(N / N^{\prime}\right)$ is finitely generated, this is not possible.

PROPOSITION 1.5. Let $R$ and $S$ be noetherian $k$-algebras, let ${ }_{S} M_{R}$ be a bimodule that is finitely generated on both sides, let $\mathrm{GK}(M)<\infty$, and assume that $M_{R}$ is equidimensional. If $M$ has a full right standard prime bi-factor series of length $n$, then $\operatorname{r.stL}(X) \leq n$ for any sub-bimodule $X$ of $M$.

Proof. Proceed by induction on $n$, the case $n=1$ being covered by Lemma 1.4. Let $n>1$, and assume that the claim has been established for all $m<n$. Let $0=N_{0} \subset N_{1} \subset$ $\ldots \subset N_{n-1}=N \subset M$ be a full right standard prime bi-factor series of $M$, and let $0=X_{0} \subset X_{1} \subset \ldots \subset X_{p-1} \subset X_{p}=X$ be a right standard prime bi-factor series of $X$. We wish to show that $p \leq n$. The distinct terms of the series $\left\{N \cap X_{i}\right\}$ form a right standard prime bi-factor series of $N \cap X$. If all $N \cap X_{i}, 0 \leq i \leq p$, are distinct, then $p \leq n-1<n$, by induction. Otherwise, let $i$ be the largest index such that $N \cap X_{i}=N \cap X_{i-1}$. Then $N+X_{i} / N+X_{i-1} \simeq X_{i} / X_{i-1}$. Set $P=r(M / N)=\operatorname{Ass}(M / N)_{R}$ and $Q=r\left(X_{i} / X_{i-1}\right)=$ Ass $\left(X_{i} / X_{i-1}\right)_{R}$. Both $P$ and $Q$ are standard primes of $M_{R}$, so $\mathrm{GK}(R / P)=\mathrm{GK}(R / Q)$ by equidimensionality. Assume that $N+X_{i-1} \supsetneq N$. Then $M / N+X_{i-1}$ and hence $N+X_{i} / N+$ $X_{i-1}$ is $\mathscr{C}(P)$-torsion by Lemma $1.4(\mathrm{i})$, so

$$
\mathrm{GK}(R / P)>\mathrm{GK}\left(N+X_{i} / N+X_{i-1}\right)=\mathrm{GK}\left(X_{i} / X_{i-1}\right)=\mathrm{GK}(R / Q),
$$

an obvious contradiction. It follows that $X_{i-1} \subseteq N$, and hence that

$$
0 \subset X_{1} \subset \ldots \subset X_{i-1}=N \cap X_{i-1}=N \cap X_{i} \subset N \cap X_{i+1} \subset \ldots \subset N \cap X_{p}=N \cap X
$$


is a right standard prime bi-factor series of the sub-bimodule $N \cap X$ of $N$. Its length is $(i-1)+(p-i)=p-1$. By induction, $p-1 \leq n-1$, hence $p \leq n$.

THEOREM 1.6. Let $R$ and $S$ be noetherian $k$-algebras, let ${ }_{s} M_{R}$ be a bimodule that is finitely generated on both sides, and let $\mathrm{GK}(M)<\infty$. Then:

(i) any two full right standard prime bi-factor series of $M$ have the same length r.stL $(M)$;

(ii) a full right standard prime bi-factor series of $M$ is also a full left standard prime bi-factor series;

(iii) $\operatorname{r.stL}(M)=1 . s t L(M)$.

Proof. (i) Since any standard prime factor series of $M_{R}$ passes through the jumping points $J_{i}$, and hence each of the bimodules $J_{i} / J_{i-1}$ satisfies the claim by Proposition 1.5, this is clear.

(ii) It is obviously enough to show that $\operatorname{r.stL}(M)=1$ implies l.stL $(M)=1$. Thus, let $P=r_{R}(M)=\operatorname{Ass}(M)_{R}$, and let $0=C_{0} \subset C_{1} \subset \ldots \subset C_{n-1} \subset C_{n}=M$ be a left standard prime bi-factor series of $M$ with $l_{S}\left(C_{i} / C_{i-1}\right)=\operatorname{Ass}_{S}\left(C_{i} / C_{i-1}\right)=Q_{i} \in \operatorname{spec}(S)$. Assume that $n>1$. Since $M / C_{n-1}$ is $\mathscr{C}(P)$-torsion as a right $R$-module by Lemma 1.4, it follows from [6, 5.3 and 5.4] that $\mathrm{GK}\left(S / Q_{n}\right)=\mathrm{GK}\left(M / C_{n-1}\right)<\mathrm{GK}(R / P)$. On the other hand, $\operatorname{GK}\left(S / Q_{1}\right)=$ $\mathrm{GK}\left(C_{1}\right)=\mathrm{GK}(R / P)$, and $\mathrm{GK}\left(S / Q_{1}\right) \leq \mathrm{GK}\left(S / Q_{n}\right)$, since the sequence $\left\{C_{i}\right\}$ is standard. This contradiction shows that l.stL $(M)=1$.

(iii) This is an immediate consequence of (i) and (ii).

Henceforth, the common value of $\operatorname{l.stL}(M)$ and $\operatorname{r.stL}(M)$ will be denoted by $\operatorname{stL}(M)$ and will be referred to as the standard length. Note that the preceding theorem also shows that the jumping points of any standard prime factor series of $M_{R}$ coincide with the jumping points of any standard prime factor series of ${ }_{s} M$. Thus, in particular, $M_{R}$ is equidimensional if and only if ${ }_{s} M$ is equidimensional. Furthermore,

$$
\left\{\mathrm{GK}(R / P) \mid P \in \mathrm{St}\left(M_{R}\right)\right\}=\left\{\mathrm{GK}(S / Q) \mid Q \in \mathrm{St}\left({ }_{S} M\right)\right\} .
$$

2. Multiplicities of standard primes. Given a right standard prime bi-factor series $\mathscr{S}=\left\{N_{i}\right\}$ of a bimodule ${ }_{s} M_{R}$, the multiplicity $\mu_{S(}\left(P, M_{R}\right)$ of the prime $P \in \operatorname{St}\left(M_{R}\right)$ is the number of times $P$ occurs in $\mathscr{S}$, that is, the number of times one has $P=r\left(N_{i} / N_{i-1}\right)=$ $\operatorname{Ass}\left(N_{i} / N_{i-1}\right)_{R}$. As these series may have different lengths (see [7, p.172]), in general one has to expect $\mu_{\mathscr{S}}\left(P, M_{R}\right) \neq \mu_{\mathscr{T}}\left(P, M_{R}\right)$ for two distinct standard series $\mathscr{S}$ and $\mathscr{T}$. However, if $\mathscr{S}$ and $\mathscr{T}$ are full right standard prime bi-factor series of $M$, then the result below shows that the multiplicities of any standard prime in either series are the same, allowing us to speak simply of the multiplicity $\mu\left(P, M_{R}\right)$ of $P$ in $M$, without having to refer to a particular series.

THEOREM 2.1. Let $R$ and $S$ be noetherian $k$-algebras, let ${ }_{s} M_{R}$ be a bimodule that is finitely generated on both sides and has finite $G K$-dimension, and let $\mathscr{S}$ and $\mathcal{T}$ be two full right standard prime bi-factor series of $M$. Then $\mu_{S(}\left(P, M_{R}\right)=\mu_{\mathcal{F}}\left(P, M_{R}\right)$ for all $P \in$ $\operatorname{St}\left(M_{R}\right)$.

Proof. Since all appearances of $P$ occur between two adjacent jumping points, we may assume that $M$ is equidimensional. We proceed by induction on $n=\operatorname{stL}(M)$, the case $n=1$ being trivial. Assume that the result holds for any bimodule ${ }_{s} X_{R}$ with $\operatorname{stL}(X)<n$. 
Let $\mathscr{S}=\left\{C_{i}\right\}$ and $\mathscr{T}=\left\{B_{i}\right\}$ be the given series. Since $\operatorname{stL}\left(B_{n-1}\right)=n-1$, and since the distinct terms of the series $\left\{C_{i} \cap B_{n-1}\right\}, 0 \leq i \leq n$ form a right standard prime bi-factor series of $B_{n-1}$, we must have $C_{i} \cap B_{n-1}=C_{i-1} \cap B_{n-1}$ for at least one index $i$. Let $i$ be the greatest such index, and set $P=r_{R}\left(C_{i} / C_{i-1}\right)=\operatorname{Ass}\left(C_{i} / C_{i-1}\right)_{R}$. Since

$$
B_{n-1}+C_{i} / B_{n-1}+C_{i-1} \simeq C_{i} / C_{i-1},
$$

it follows that

$$
P_{n}=r\left(M / B_{n-1}\right) \subseteq r\left(B_{n-1}+C_{i} / B_{n-1}+C_{i-1}\right)=r\left(C_{i} / C_{i-1}\right)=P,
$$

whence $P=P_{n}$, by equidimensionality. If $B_{n-1} \varsubsetneqq B_{n-1}+C_{i-1}$, then $C_{i} / C_{i-1}$ would be $\mathscr{C}(P)$-torsion by Lemma 1.4. As this is not the case, $C_{i-1} \subseteq B_{n-1}$, which proves that $C_{j} \cap B_{n-1}=C_{j-1} \cap B_{n-1}$ occurs only for $j=i$. Thus, the distinct terms $C_{j} \cap B_{n-1}$ form a full right standard prime factor series of $B_{n-1}$ of length $n-1$. Consequently, $\mu_{s d}(P, M)-$ $1=\mu\left(P, B_{n-1}\right)$, by induction. Since obviously $\mu_{\mathscr{T}}(P, M)=\mu\left(P, B_{n-1}\right)+1$, it follows that $\mu_{\mathscr{S}}(P, M)=\mu_{\mathscr{T}}(P, M)$. If $Q \in \operatorname{St}\left(M_{R}\right), Q \neq P$, then $Q$ occurs in $\mathscr{S}$ as $Q=r\left(C_{h} / C_{h-1}\right)=$ Ass $\left(C_{h} / C_{h-1}\right)_{R}$ with $h \neq i$ if and only if $Q$ occurs in the series $\left\{C_{j} \cap B_{n-1}, j \neq i\right\}$ of $B_{n-1}$ as $Q=r\left(C_{h} \cap B_{n-1} / C_{h-1} \cap B_{n-1}\right)=\operatorname{Ass}\left(C_{h} \cap B_{n-1} / C_{h-1} \cap B_{n-1}\right)_{R}, \quad$ so $\quad \mu_{f}(Q, M)=$ $\mu\left(Q, B_{n-1}\right)$. Since $Q \neq P=r\left(M / B_{n-1}\right)$, we also have that $\mu\left(Q, B_{n-1}\right)=\mu_{\mathscr{T}}(Q, M)$, so $\mu_{\mathscr{S}}(Q, M)=\mu_{\mathscr{T}}(Q, M)$.

Considering the special case $R=S$ and a bimodule $M$ that has a prime ideal $P$ belonging to both $\operatorname{St}\left(M_{R}\right)$ and $\left.\mathrm{St}_{R} M\right)$, the right and left multiplicities of $P$ need not be the same. The following example demonstrates this, it even satisfies $\operatorname{St}\left(M_{R}\right)=\operatorname{St}\left({ }_{R} M\right)$.

EXAMPLE 2.2. Let $M=R$, the ring of upper triangular $2 \times 2$ matrices over $k$. Let

$$
P=\left[\begin{array}{ll}
0 & k \\
0 & k
\end{array}\right], \quad Q=\left[\begin{array}{cc}
k & k \\
0 & 0
\end{array}\right], \quad \text { and } N=P \cap Q=\left[\begin{array}{ll}
0 & k \\
0 & 0
\end{array}\right] .
$$

It is easy to see that $0 \subset N \subset Q \subset R=M$ is a full left and right standard prime bi-factor series of $M$ with $Q=r(N)=\operatorname{Ass}(N)_{R}, P=r(Q / N)=\operatorname{Ass}(Q / N)_{R}$, and $Q=r(R / Q)=$ $\operatorname{Ass}(R / Q)_{R}$, so that $\operatorname{St}\left(M_{R}\right)=\{P, Q\}$. As $P=l(N)=\operatorname{Ass}_{R}(N), P=l(Q / N)=\operatorname{Ass}_{R}(Q / N)$, and $Q=l(R / Q)=\operatorname{Ass}_{R}(R / Q)$, we also have that $\left.\operatorname{St}_{R} M\right)=\{P, Q\}$. However, $\mu\left(P, M_{R}\right)=$ 1 , yet $\mu\left(P,{ }_{R} M\right)=2$. Similarly, $\mu\left(Q, M_{R}\right)=2$, but $\mu\left(Q,{ }_{R} M\right)=1$.

3. Lying over. Let $R \subset S$ be a right finite extension of noetherian $k$-algebras, that is, $R$ is a noetherian subalgebra of the noetherian algebra $S$, and $S$ is finitely generated as a right $R$-module. In this section, standard primes are used to investigate the relationship between $\operatorname{spec}(R)$ and $\operatorname{spec}(S)$, where $R$ is assumed to have finite GK-dimension. Note that in this set-up, $\mathrm{GK}(S)=\mathrm{GK}(R)$ by [6, Proposition 5.5].

Definition. A prime ideal $Q$ of $S$ lies over the prime ideal $P$ of $R$ if $P$ is minimal over $Q \cap R$. A prime $P$ of $R$ is said to have lying over, or to have $L O$, for short, if at least one prime of $S$ lies over $P$; if, in this case, only finitely many primes of $S$ lie over $P$, then $P$ is said to have finite $L O$.

Due to Lenagan (see [8, Theorem 2.1]), if $S_{R}$ is finitely generated and free, then every prime of $R$ has LO. The following example shows that this is no longer the case when $S_{R}$ 
is merely projective. Although the example has been known for some time, the fact that it has GK-dimension 2 does not seem to appear in the literature, so we include the simple calculation. The fact that lying over may fail already for GK-dimension 2 is noteworthy in view of the fact that if $R$ is an affine noetherian $k$-algebra of GK-dimension 1, then $R$ is PI by [13], so it satisfies the second layer condition, so lying over holds for all primes of $R$ by [9, Theorem 4.6].

EXAMPLE 3.1 (Lorenz [11], Hodges and Osterburg [3]). Let $k$ be a field of characteristic 2, containing a nonzero element $\lambda$ that is not a root of unity. Let $S=k\left\{x, x^{-1}, y, y^{-1}\right\} /(x y-\lambda y x)$. By [11, Example 1.8], $S$ is a simple noetherian domain. If $R=S^{\sigma}$, the subalgebra fixed by the automorphism $\sigma$ of $S$ defined via $\sigma(x)=x^{-1}$, $\sigma(y)=y^{-1}$, then both ${ }_{R} S$ and $S_{R}$ are finitely generated, indecomposable, and projective by [3, Theorem 1], and $R$ is noetherian by [3, Theorem 5]. The ideal $P=\{s+\sigma(s) \mid s \in S\}$ is the only nonzero proper ideal of the domain $R$, and since $S$ is simple, LO does not hold for $P$. It remains to show that $\mathrm{GK}(R)=\mathrm{GK}(S)=2$. Identifying $x$ and $y$ with their canonical images in $S$, the elements $x^{i} y^{j}$ with integers $i$ and $j$ form a basis for $S$ over $k$. The subspace $V=k \oplus k x \oplus k x^{-1} \oplus k y \oplus k y^{-1}$ is a generating subspace for the algebra $S$. As $V^{n}=V^{n-1} \oplus \sum_{|i|+|j|=n} k x^{i} y^{j}, \operatorname{dim}_{k}\left(V^{n}\right)-\operatorname{dim}_{k}\left(V^{n-1}\right)=4 n$ for $n \geq 1$. Therefore, $\operatorname{dim}_{k}\left(V^{n}\right)=$ $1+\sum_{j=1}^{n} 4 j=1+2(n+1) n$, whence $\mathrm{GK}(S)=2$.

Being thus faced with the fact that lying over does not universally hold for finite extensions of noetherian algebras with finite GK-dimension, one is led to search for criteria that will allow one to recognize when a given prime ideal of $R$ has LO. Since by [5, Theorem 3.3] a prime $P$ is a standard prime of a right $R$-module $M$ if and only if $P$ is minimal over the annihilator of some nonzero submodule of $M$, a connection between lying over and standard primes seems almost natural. Indeed, $Q \in \operatorname{spec}(S)$ lies over $P \in \operatorname{spec}(R)$ if and only if $P \in \mathrm{St}(S / Q)_{R}=\mathrm{St}(R / Q \cap R)_{R}$, as is easily seen from the proof of [9, Lemma 3.2]. Generalizing this somewhat, we have the following result.

Proposition 3.2. Let $R \subset S$ be a right finite extension of noetherian $k$-algebras with finite $G K$-dimension. Then the following statements are equivalent for an ideal I of $S$.

(1) $S / I$ is equidimensional as an $S$-module

(2) (a) $R / I \cap R$ is equidimensional as an $R$-module.

(b) $\operatorname{St}(R / I \cap R)_{R}=\operatorname{St}(S / I)_{R}=\{P \in \operatorname{spec}(R) \mid P$ minimal over $I \cap R\}$.

Proof. Note that in view of Theorem 1.6 one does not have to distinguish between right and left equidimensional for an $S$ - $R$-bimodule that is finitely generated on both sides. Let $l=B_{0} \subset B_{1} \subset \ldots \subset B_{i-1} \subset B_{i} \subset \ldots \subset B_{n}=S$ be a full standard prime bi-factor series of ${ }_{S}(S / I)_{R}$ with $Q_{i}=l_{S}\left(B_{i} / B_{i-1}\right)=\operatorname{Ass}_{S}\left(B_{i} / B_{i-1}\right)$, and $P_{i}=r_{R}\left(B_{i} / B_{i-1}\right)=$ $\operatorname{Ass}\left(B_{i} / B_{i-1}\right)_{R}$.

$(1) \Rightarrow(2)$. Since $\mathrm{GK}\left(S / Q_{i}\right)=\mathrm{GK}\left(R / P_{i}\right)$ for $1 \leq i \leq n,(S / I)_{R}$ is equidimensional, so $R / I \cap R=R+I / I \subseteq S / I$ is equidimensional, giving (2)(a). For (2)(b), observe that any prime that is minimal over $I \cap R$ belongs to $\operatorname{St}(R / I \cap R)_{R}$ by [5, Theorem 3.3]. Also, $\operatorname{St}(R / I \cap R)_{R} \subseteq \operatorname{St}(S / I)_{R}$ by [5, Corollary 3.5]. Thus, in order to establish equality of the three sets in (2)(b), let $P \in \mathrm{St}(S / I)_{R}$. As $(S / I)_{R}$ is equidimensional, $P$ is minimal over $r_{R}(S / I)=r_{S}(S / I) \cap R=I \cap R$ by Lemma 1.1. 
(2) $\Rightarrow(1)$. Since $\left\{P_{1}, \ldots, P_{n}\right\}=\mathrm{St}(S / I)_{R}=\operatorname{St}(R / I \cap R)_{R}$, and since $R / I \cap R$ is equidimensional, it follows that $\mathrm{GK}\left(S / Q_{i}\right)=\mathrm{GK}\left(R / P_{i}\right)=\mathrm{GK}\left(R / P_{j}\right)=\mathrm{GK}\left(S / Q_{j}\right)$ for all $1 \leq$ $i, j \leq n$, showing that $S / I$ is equidimensional.

We proceed to relate the standard primes of $S$ to those of $R$. In order to obtain a criterion for lying over, it is convenient to examine an $S$-S-bimodule $M$ such that ${ }_{S} M_{R}$ is finitely generated on each side.

THEOREM 3.3. Let $R \subset S$ be an extension (not necessarily finite) of noetherian $k$-algebras with finite $G K$-dimension. Let $M$ be an $S$-S-bimodule such that ${ }_{S} M_{R}$ is finitely generated on both sides. Then:

(a) each $Q \in \mathrm{St}\left(M_{S}\right)$ lies over some $P \in \mathrm{St}\left(M_{R}\right)$;

(b) if $Q \in \mathrm{St}\left(M_{S}\right)$ lies over $P \in \operatorname{spec}(R)$, then $P \in \mathrm{St}\left(M_{R}\right)$;

(c) for every $P \in \mathrm{St}\left(M_{R}\right)$ there exists a $Q \in \mathrm{St}\left(M_{S}\right)$ that lies over $P$.

Proof. Clearly ${ }_{s} M_{S}$ is finitely generated on both sides and $\mathrm{GK}(M)<\infty$. Using Theorem 1.6, choose a full standard prime bi-factor series of ${ }_{s} M_{S}$ :

$$
0=N_{0} \subset N_{1} \subset \ldots \subset N_{i-1} \subset N_{i} \subset \ldots \subset N_{m}=M
$$

with $Q_{i}=r_{S}\left(N_{i} / N_{i-1}\right)=\operatorname{Ass}\left(N_{i} / N_{i-1}\right)_{S}, \quad Q_{i}^{*}=l_{S}\left(N_{i} / N_{i-1}\right)=\operatorname{Ass}_{S}\left(N_{i} / N_{i-1}\right)$. Refine this series by choosing a full right standard prime bi-factor series for each ${ }_{s}\left(N_{i} / N_{i-1}\right)_{R}$ :

$$
N_{i-1}=C_{i, 0} \subset C_{i, 1} \subset \ldots \subset C_{i, j-1} \subset C_{i, j} \subset \ldots \subset C_{i, n(i)}=N_{i}
$$

with $r_{R}\left(C_{i, j} / C_{i, j-1}\right)=\operatorname{Ass}\left(C_{i, j} / C_{i, j-1}\right)_{R}=P_{i, j}$. By Theorem 1.6, this is also a full left standard prime bi-factor series for ${ }_{S}\left(N_{i} / N_{i-1}\right)_{R}$. Since $\mathrm{St}_{s}\left(N_{i} / N_{i-1}\right)=\left\{Q_{i}^{*}\right\}$, it follows that $l_{S}\left(C_{i j} / C_{i, j-1}\right)=\operatorname{Ass}_{S}\left(C_{i j} / C_{i,-1}\right)=Q_{i}^{*}$ for all $1 \leq j \leq n(i)$, and hence that $\mathrm{GK}\left(S / Q_{i}\right)=$ $\mathrm{GK}\left(S / Q_{i}^{*}\right)=\mathrm{GK}\left(R / P_{i, j}\right)$ for all $1 \leq i \leq m, 1 \leq j \leq n(i)$ by [6,5.3 and 5.4]. Consequently, the combined series $\left\{C_{i, j}\right\}$ is a full standard prime bi-factor series of ${ }_{s} M_{R}$. As ${ }_{s}\left(N_{i} / N_{i-1}\right)$ is finitely generated, the right $R$-module $R / Q_{i} \cap R=R / r_{R}\left(N_{i} / N_{i-1}\right)$ embeds in a direct sum $D_{R}$ of finitely many copies of $\left(N_{i} / N_{i-1}\right)_{R}$. Note that $\operatorname{St}\left(D_{R}\right)=\operatorname{St}\left(N_{i} / N_{i-1}\right)_{R}$. Now let $Q \in \operatorname{St}\left(M_{S}\right)$, so $Q=Q_{i}$ for some $i$. Let $P$ be minimal over $Q_{i} \cap R$. Since $S / Q_{i}$ is equidimensional, Proposition 3.2 implies that

$$
P \in \mathrm{St}\left(R / Q_{i} \cap R\right)_{R} \subseteq \mathrm{St}\left(D_{R}\right)=\mathrm{St}\left(N_{i} / N_{i-1}\right)_{R}=\left\{P_{i j} \mid 1 \leq j \leq n(i)\right\},
$$

and since the latter set is a subset of $\operatorname{St}\left(M_{R}\right)$, this proves (a) and (b). Finally, let $P \in \mathrm{St}\left(M_{R}\right)$, so $P=P_{i, j}$ for some $(i, j)$. As $\left(N_{i} / N_{i-1}\right)_{R}$ is equidimensional, Lemma 1.1 shows that $P$ is minimal over $r_{R}\left(N_{i} / N_{i-1}\right)=Q_{i} \cap R$, proving (c).

In view of part (b) of the preceding result, the question arises whether $Q \in \operatorname{St}\left(M_{S}\right)$ when $Q$ lies over $P \in \operatorname{St}\left(M_{R}\right)$. However, this is generally not the case.

Example 3.4 (see $[5$, p. 224]). Let $k$ be a field, let $F=k[x] /(x)$, considered as a $k[x]-k$-bimodule, and set

$$
S=\left[\begin{array}{cc}
k[x] & F \\
0 & k
\end{array}\right], \quad R=\left\{\left[\begin{array}{cc}
f(x) & 0 \\
0 & f(0)
\end{array}\right] \mid f(x) \in k[x]\right\} \simeq k[x] .
$$


Then $R$ is a noetherian subalgebra of the noetherian $k$-algebra $S, S_{R}$ is finitely generated by $\left[\begin{array}{ll}1 & 0 \\ 0 & 0\end{array}\right],\left[\begin{array}{cc}0 & 1+(x) \\ 0 & 0\end{array}\right]$, and $\left[\begin{array}{ll}0 & 0 \\ 0 & 1\end{array}\right]$, and $\mathrm{GK}(S)=\mathrm{GK}(R)=1$. Let $N=\left[\begin{array}{ll}0 & F \\ 0 & 0\end{array}\right]$, $Q_{1}=\left[\begin{array}{ll}0 & F \\ 0 & k\end{array}\right] \varsubsetneqq Q_{3}=\left[\begin{array}{cc}x k[x] & F \\ 0 & k\end{array}\right], Q_{2}=\left[\begin{array}{cc}k[x] & F \\ 0 & 0\end{array}\right]$. Then $0 \subset N \subset Q_{1} \subset S$ is a full right standard prime bi-factor series of ${ }_{s} S_{S}$ with $r_{s}(N)=\operatorname{Ass}(N)_{s}=Q_{2}, \quad r_{s}\left(Q_{1} / N\right)=$ $\operatorname{Ass}\left(Q_{1} / N\right)_{S}=Q_{2}, r_{S}\left(S / Q_{1}\right)=\operatorname{Ass}\left(S / Q_{1}\right)_{S}=Q_{1}$. This series is also easily seen to be a full right standard prime bi-factor series of $s_{R}$ with $r_{R}(N)=\operatorname{Ass}(N)_{R}=P_{1}=\left[\begin{array}{cc}x k[x] & 0 \\ 0 & 0\end{array}\right]$, $r_{R}\left(Q_{1} / N\right)=\operatorname{Ass}\left(Q_{1} / N\right)_{R}=P_{1}, \quad r_{R}\left(S / Q_{1}\right)=\operatorname{Ass}\left(S / Q_{1}\right)_{R}=P_{2}=0 . \quad$ Now observe that $Q_{3} \cap R=P_{1}$, so $Q_{3}$ lies over $P_{1} \in \mathrm{St}\left(S_{R}\right)$, yet $Q_{3} \notin \operatorname{St}\left(S_{S}\right)=\left\{Q_{1}, Q_{2}\right\}$.

THEOREM 3.5. Let $R \subset S$ be a right finite extension of noetherian $k$-algebras with finite $G K$-dimension. The following statements are equivalent for a prime ideal $P$ of $R$.

(1) $P$ has $L O$.

(2) $I P \cap R \subseteq P$ for some ideal I of $S$ with $\mathrm{GK}(S / I) \leq \mathrm{GK}(R / P)$.

(3) $P \in \mathrm{St}(R / I \cap R)_{R}$ for some ideal I of $S$.

(4) $P \in \mathrm{St}(S / I)_{R}$ for some ideal I of $S$.

Proof. (1) $\Rightarrow$ (2). If $P$ is minimal over $Q \cap R$ for some $Q \in \operatorname{Spec}(S)$, then $P \in$ $\operatorname{St}(R / Q \cap R)_{R} \subseteq \operatorname{St}(S / Q)_{R}$. Looking at a full right standard prime bi-factor series of ${ }_{s}(S / Q)_{R}$, we conclude that $\mathrm{GK}(S / Q)=\mathrm{GK}(R / P)$. Also $Q P \cap R \subseteq Q \cap R \subseteq P$.

(2) $\Rightarrow$ (3). Since $(I \cap R)(S P \cap R) \subseteq I P \cap R \subseteq P$, either $I \cap R \subseteq P$ or $S P \cap R \subseteq P$. In the first case, it follows from $\mathrm{GK}(R / P) \leq \mathrm{GK}(R / I \cap R)=\mathrm{GK}(S / I) \leq \mathrm{GK}(S / I) \leq$ $\mathrm{GK}(R / P)$ that $P$ is minimal over $I \cap R$, whence $P \in \mathrm{St}(R / I \cap R)_{R}$. In the second case, $P=S P \cap R=r_{R}(S / S P)$, so if $J=l_{S}(S / S P)$, then $\mathrm{GK}(R / J \cap R)=\mathrm{GK}(S / J)=\mathrm{GK}(S / S P)=$ $\mathrm{GK}(R / P)$ by [6,5.3 and 5.4]. As $J \cap R=J S \cap R \subseteq S P \cap R=P, P$ is thus minimal over $J \cap R$, whence $P \in \mathrm{St}(R / J \cap R)_{R}$.

(3) $\Rightarrow(4)$. This is clear since $\mathrm{St}(R / I \cap R)_{R}=\mathrm{St}(R+I / I)_{R} \subseteq \mathrm{St}(S / I)_{R}$.

(4) $\Rightarrow(1)$. The $S$-S -bimodule $M=S / I$ satisfies the hypotheses of Theorem 3.3, so the claim follows from part (c) of that result.

As a special case of the preceding theorem, $P \in \operatorname{spec}(R)$ has LO whenever $S P \cap R=P$. (Note that this also follows as in the proof of $[8,2.1]$.) In the next section we shall see that this condition characterizes a special case of lying over, called "lying directly over" by Goodearl and Letzter [1].

\section{Lying directly over.}

Definition. Let $R \subset S$ be rings. A prime ideal $Q$ of $S$ lies right (left) directly over the prime ideal $P$ of $R$ if

(a) $Q$ lies over $P$,

(b) $P \in$ annspec $(S / Q)_{R}\left(\operatorname{annspec}_{R}(S / Q)\right)$, that is, $P$ is the right (left) annihilator of a nonzero right (left) $R$-submodule of $S / Q$.

It will be shown below (Example 4.9) that the distinction between right and left in 
the above definition is necessary in general. The following result shows that for the situation discussed in this paper, condition (a) follows from (b). For this, see also [1, Lemma 5.3].

PROPOSITION 4.1. Let $R \subset S$ be a right finite extension of noetherian $k$-algebras with finite $G K$-dimension. Let $P \in \operatorname{spec}(R), Q \in \operatorname{spec}(S)$. Then:

(i) if $P \in$ annspec $(S / Q)_{R}$, then $Q$ lies over $P=P S \cap R$;

(ii) if $P \in \operatorname{annspec}_{R}(S / Q)$, then $Q$ lies over $P=S P \cap R$ and $Q$ is minimal over $l_{S}(S / S P)$.

Proof. (i) Assume that $P=r_{R}(X / Q)$ for a nonzero right $R$-submodule $X / Q$ of $S / Q$. By [5, Theorem 3.3], $P \in \mathrm{St}(S / Q)_{R}$. As $S / Q$ is equidimensional, $P$ is minimal over $Q \cap R$ by Proposition 3.2. Note that $P \subseteq P S \cap R$ in general. Since $X(P S \cap R) \subseteq X P S \subseteq Q S=Q$, $P S \cap R \subseteq r_{R}(X / Q)=P$.

(ii) If $P=l_{R}(X / Q), 0 \neq X / Q \subseteq{ }_{R}(S / Q)$, then $P=S P \cap R$, analogous to what has been shown in (i) on the right hand side. Now $X$ can be assumed to be a right ideal of $S$, so it follows from $[6,5.3]$ that $\mathrm{GK}(R / P)=\mathrm{GK}_{R}(X / Q) \leq \mathrm{GK}(X / Q)_{S} \leq \mathrm{GK}(S / Q)$. Set $I=l_{S}(S / S P)$, and note that $P=S P \cap R=r_{R}(S / S P)$. By [6, 5.3 and 5.4], it follows that $\mathrm{GK}(R / P)=\mathrm{GK}(S / S P)=\mathrm{GK}(S / I)$. As $I S X \subseteq S P X \subseteq Q$ and $S X \nsubseteq Q, I \subseteq Q$ follows. Consequently, $\mathrm{GK}(R / P) \leq \mathrm{GK}(S / Q) \leq \mathrm{GK}(S / I)=\mathrm{GK}(R / P)$, so equality holds throughout, proving that $Q$ is minimal over $I$. Since $\mathrm{GK}(R / P)=\mathrm{GK}(S / Q)=\mathrm{GK}(R / Q \cap R)$, and since obviously $Q \cap R \subseteq P, P$ is minimal over $Q \cap R$.

As a consequence of 4.1(ii) we have the following result which also appears in [10, Proposition 4.2(iii)] under the additional assumption that ${ }_{R} S$ is also finitely generated.

COROLLARY 4.2. Let $R \subset S$ be a right finite extension of noetherian $k$-algebras with finite $G K$-dimension. Then at most finitely many prime ideals of $S$ can lie left directly over a given prime of $R$.

Proof. If $Q \in \operatorname{spec}(S)$ lies left directly over $P \in \operatorname{spec}(R)$, then $Q$ is minimal over $I=l_{S}(S / S P)$ by 4.1 (ii). As $S / I$ is noetherian, the claim follows.

Although Letzter [10] has recently made considerable progress, it is still not known whether only a finite number of prime ideals of $S$ can lie over a given prime of $R$. What Letzter has shown for a right finite extension of noetherian rings is that for a given prime $P$ of $R$ at most finitely many prime ideals $Q$ of $S$ can lie over $P$ for which the Goldie rank of $S / Q$ is a prescribed positive integer. This shows that at most countably many primes of $S$ can lie over $P$, a result which we can obtain with a different proof for the special case that $R \subset S$ is a right finite extension of noetherian algebras with finite GK-dimension.

COROLlaRY 4.3. Let $R \in S$ be a right finite extension of noetherian $k$-algebras with finite $G K$-dimension. Let $P \in \operatorname{spec}(R)$. Then the set of prime ideals of $S$ that lie over $P$ is at most countable.

Proof. Let $\left\{Q_{i} \in \operatorname{spec}(S) \mid i \in I\right\}$ be the set in question, and assume that $I$ is not empty. For each $i \in I, P \in \mathrm{St}_{R}\left(R / Q_{i} \cap R\right)$, so by [5, Theorem 3.2] there exists a prime ideal $P_{i}$ in the clique of $P$ such that $P_{i}$ is the left annihilator of a nonzero submodule of ${ }_{R}\left(R / Q_{i} \cap R\right)$. As $R / Q_{i} \cap R=R+Q_{i} / Q_{i} \subseteq S / Q_{i}$ as left $R$-modules, $Q_{i}$ thus lies left directly over $P_{i}$. Since cliques in a noetherian ring are countable by a theorem of Stafford 
(see $[2,14.23]$ ), the preceding corollary shows that $I$ is the union of countably many finite sets, hence countable.

The next result shows how to recognize whether a prime ideal $Q$ of $S$ lies left directly over a prime ideal $P$ of $R$. It should be compared with [1,5.5] and [10, 4.1 and 4.2].

Proposition 4.4. Let $R \subset S$ be a right finite extension of noetherian $k$-algebras with finite $G K$-dimension. Let $P \in \operatorname{spec}(R), Q \in \operatorname{spec}(S)$. Then the following statements are equivalent.

(1) $\mathrm{GK}(S / Q)=\mathrm{GK}(R / P)$, and there exists an $S$-R-bimodule factor $E$ of ${ }_{S}(S / Q)_{R}$ with $r_{R}(E)=P$, such that $E$ is torsionfree as a right $R / P$-module.

(2) $\mathrm{GK}(S / Q)=\mathrm{GK}(R / P)$, and there exists an $S$-R-bimodule factor $F$ of $S(S / S P)_{R}$ with $l_{S}(F)=Q$, such that $F$ is torsionfree as a left $S / Q$-module.

(3) $Q$ lies left directly over $P$.

Proof. (1) $\Rightarrow(2)$. Let $B / Q$ be the kernel of the canonical $S$-R-bimodule homomorphism $S / Q \rightarrow E$. Then $S P \subseteq B$. Also, $Q \subseteq l_{S}(E)$, and since $E$ is finitely generated on each side and torsionfree as a right $R / P$-module, it follows from $\mathrm{GK}(S / Q)=\mathrm{GK}(R / P)$, that $Q=l_{S}(E)$ and that $E$ is torsionfree as a left $S / Q$-module. Thus one can set $F=S / B \simeq E$.

(2) $\Rightarrow$ (3). Let $F \simeq S / B, S P \subseteq{ }_{s} B_{R} \subseteq S$. Then $P \subseteq S P \cap R \subseteq B \cap R$. As $S / B$ is an $S / Q-R / B \cap R$-bimodule, finitely generated and faithful on each side, it follows that $\mathrm{GK}(S / Q)=\mathrm{GK}(S / B)=\mathrm{GK}(R / B \cap R) \leq \mathrm{GK}(R / P)=\mathrm{GK}(S / Q)$, so $\mathrm{GK}(R / B \cap R)=$ $\mathrm{GK}(R / P)$, so the ideal $B \cap R$ of $R$ cannot properly contain $P$. Thus $R / P=R / B \cap R \subseteq$ $S / B$ as left $R$-modules. Now, $S / B$, being a finitely generated and torsionfree left $S / Q$-module, embeds in a finite direct sum of copies of $S / Q$ as a left $S$-module (see [2, 6.19]), and hence also as a left $R$-module. It follows that $R / P$ embeds in a finite direct sum of copies of ${ }_{R}(S / Q)$, so $P \in \operatorname{Ass}_{R}(S / Q)$, so by 4.1(ii), $Q$ lies directly over $P$.

(3) $\Rightarrow(1)$. If $Q$ lies left directly over $P$, then $\mathrm{GK}(S / Q)=\mathrm{GK}(R / P)$ by Proposition 3.2. Let $P=l_{R}(X / Q)$ for some left $R$-submodule $X / Q \neq 0$ of $S / Q$, where $X$ may be assumed to be a right ideal of $S$. Let $A=l_{S}(X / Q) \supseteq Q$, and note that $A$ is an $S$ - $R$-sub-bimodule of $S$. Since $X / Q$ is a finitely generated right ideal of $S / Q, S / A$ embeds in a finite direct sum of copies of $S / Q$ as a left $S$-module, showing that $Q=l_{S}(S / A)$, and that $S / A$ is torsionfree as a left $S / Q$-module. Obviously, $P=r_{R}(S / A)$, and $(S / A)_{R / P}$ is torsionfree since $s / Q(S / A)_{R / P}$ is finitely generated on both sides and torsionfree on the left, and since $\mathrm{GK}(R / P)=\mathrm{GK}(S / Q)$. Setting $E=S / A$ gives (1).

It has already been noted that if some prime ideal of $S$ lies left directly over $P \in \operatorname{spec}(R)$, then $S P \cap R=P$. It turns out, that this equality is sufficient for $P$ to have a prime of $S$ lying left directly over it.

THEOREM 4.5. Let $R \subset S$ be a right finite extension of noetherian $k$-algebras with finite $G K$-dimension. The following statements are equivalent for a prime ideal $P$ of $R$.

(1) $P=l_{R}(X)$ for some left $R$-submodule $X$ of a left $S$-module.

(2) $P=L \cap R$ for some left ideal $L$ of $S$.

(3) $P=S P \cap R$.

(4) There exists a prime ideal $Q$ of $S$ that lies left directly over $P$. 
Proof. (1) $\Rightarrow(2)$. Set $L=l_{S}(X)$.

(2) $\Rightarrow$ (3). As $P \subseteq L, S P \cap R \subseteq L \cap R=P$.

(3) $\Rightarrow(4)$. As $P=S P \cap R=\dot{r}_{R}(S / S P), P \in \mathrm{St}(S / S P)_{R}$. Let

$$
S P=B_{0} \subset B_{1} \subset \ldots \subset B_{i-1} \subset B_{i} \subset \ldots \subset B_{n-1} \subset B_{n}=S
$$

be a full standard prime bi-factor series of $s(S / S P)_{R}$ with $P_{i}=r_{R}\left(B_{i} / B_{i-1}\right)=$ $\operatorname{Ass}\left(B_{i} / B_{i-1}\right)_{R}, Q_{i}=l_{S}\left(B_{i} / B_{i-1}\right)=\operatorname{Ass}_{S}\left(B_{i} / B_{i-1}\right)$. Note that $E=S / B_{n-1}$ is an $S / Q_{n}-R / P_{n}$ module, faithful and torsionfree on either side. Note also that $\operatorname{GK}\left(S / Q_{n}\right)=$ $\mathrm{GK}\left(S / B_{n-1}\right)=\mathrm{GK}\left(R / P_{n}\right)$, so (1) will follow from 4.4 if we can establish that $P=P_{n}$. But this follows from $P=r_{R}(S / S P) \subseteq r_{R}\left(S / B_{n-1}\right)=P_{n}$, and from the fact that $\mathrm{GK}\left(R / P_{n}\right) \geq$ $\mathrm{GK}(R / P)$ due to $P \in \mathrm{St}(S / S P)_{R}$.

$(4) \Rightarrow(1)$. By definition of lying left directly over.

It has been established in [15] that the trace ideal of $S$ in $R$ plays a significant role in the interplay between properties of $R$ and $S$. Recall that the right trace ideal is $T\left(S_{R}\right)=\Sigma\left\{f(S) \mid f \in \operatorname{Hom}\left(S_{R}, R_{R}\right)\right\}$. It is proved in [14], that $P \in \operatorname{spec}(R)$ has LO if $T\left(S_{R}\right) \varsubsetneqq P$. The following result sharpens this a little.

COROLlaRY 4.6. Let $R \subset S$ be a right finite extension of noetherian $k$-algebras with finite $G K$-dimension. Let $P \in \operatorname{spec}(R)$. Then:

(a) if $T\left(S_{R}\right) \nsubseteq P$, then some prime ideal of $S$ lies left directly over $P$;

(b) if $S_{R}$ is projective and some prime of $S$ lies left directly over $P$, then $T\left(S_{R}\right) \ddagger P$.

Proof. (a) For some $f \in \operatorname{Hom}\left(S_{R}, R_{R}\right), f(S) \nsubseteq P$, so it follows from

$$
f(S)(S P \cap R)=f(S(S P \cap R)) \subseteq f(S P)=f(S) P \subseteq P
$$

that $S P \cap R \subseteq P$. Hence $S P \cap R=P$, and the claim follows from 4.5.

(b) If some prime $Q$ of $S$ lies left directly over $P$, then by Proposition 4.4 there exists an $S$-R-bimodule factor $E$ of $S / Q$ with $r_{R}(E)=P$ and $E_{R / P}$ being torsionfree. Since $E_{R / P}$ is finitely generated, $E_{R / P}$ embeds in a finite direct sum of copies of $R / P$ as a right $R$-module (see $[2,6.19]$ ). Thus there exists a nonzero right $R$-module homomorphism $g: S \rightarrow R / P$. If $S_{R}$ is projective, then there exists $f \in \operatorname{Hom}\left(S_{R}, R_{R}\right)$ with $\pi f=g$, where $\pi: R \rightarrow R / P$ is the canonical map. Obviously, $f(S) \nsubseteq P$.

As another application of Theorem 4.5 we present a further criterion for a prime ideal of $R$ to have LO. For this, we extend the notion of a standard prime to arbitrary (that is, not necessarily finitely generated) modules over a one-sided noetherian ring.

Definition. Let $R$ be a left noetherian ring, and let $M$ be a left $R$-module. A prime ideal $P$ of $R$ is said to be a standard prime of $M$, if $P$ is minimal over $l_{R}(X)$ for some finitely generated nonzero submodule $X$ of $M$. The set of standard primes of $M$ is denoted by $\operatorname{St}(M)$.

Lemma 4.7. Let $R$ be a left noetherian ring, let $M$ be a left $R$-module, and let $N$ be a submodule of $M$. Then $\operatorname{St}(N) \subseteq \operatorname{St}(M) \subseteq \operatorname{St}(N) \cup \operatorname{St}(M / N)$.

Proof. The first inclusion is trivial. Let $P \in \operatorname{St}(M)$, and let $X$ be a nonzero finitely generated submodule of $M$ such that $P$ is minimal over $l_{R}(X)$. If $X \subseteq N$, then $P \in \operatorname{St}(N)$, 
so assume that $X \nsubseteq N$. Since $l(X \cap N) l(X / X \cap N) \subseteq l(X) \subseteq P, \quad l(X \cap N) \subseteq P$ or $l(X / X \cap N) \subseteq P$. In the first case, $P$ is minimal over $l(X \cap N)$, and since $R$ is left noetherian, $X \cap N$ is finitely generated, whence $P \in \operatorname{St}(N)$. In the second case, $P$ is minimal over $l(X / X \cap N)=l(X+N / N)$, so $P \in \operatorname{St}(M / N)$.

THEOREM 4.8. Let $R \subset S$ be a right finite extension of noetherian $k$-algebras with finite $G K$-dimension. A prime ideal $P$ of $R$ has $L O$ if and only if $\left.P \in \operatorname{St}_{R} M\right)$ for some left $S$-module $M$.

Proof. If $Q \in \operatorname{spec}(S)$ lies over $P$, then $P$ is minimal over $Q \cap R=l_{R}(R / Q \cap R)=$ $l_{R}(R+Q / Q)$. As $R+Q / Q$ is a finitely generated left $R$-submodule of $S / Q$, we have that $P \in \mathrm{St}_{R}(S / Q)$. For the converse, let $P$ be minimal over $l_{R}(X), X$ a finitely generated left $R$-submodule of ${ }_{S} M$. Without loss of generality, we may assume that $M=S X$, hence that ${ }_{s} M$ is finitely generated. Now let

$$
0=B_{0} \subset B_{1} \subset \ldots \subset B_{i-1} \subset B_{i} \subset \ldots \subset B_{n}=M
$$

be a standard prime factor series of ${ }_{S} M$. As $\left.P \in \mathrm{St}_{R} M\right), P \in \mathrm{St}_{R}\left(B_{i} / B_{i-1}\right)$ for some $i$, by Lemma 4.7. Replacing $M$ by $B_{i} / B_{i-1}$, we may therefore further assume that $l_{S}(M)=$ $\operatorname{Ass}_{S}(M)=Q \in \operatorname{spec}(S)$. By [5, Theorem 3.2] there is some prime ideal $\left.P^{*} \in \operatorname{St}_{(} X\right)$ that belongs to the clique of $P$ such that $P^{*}=l_{R}(Y)$ for some nonzero submodule $Y$ of ${ }_{R} X$. Note that $\mathrm{GK}\left(R / P^{*}\right)=\mathrm{GK}(R / P)$. By Theorem 4.5, $S P^{*} \cap R=P^{*}$. Now, $P^{*}=l_{R}(Y) \supseteq$ $l_{R}(S Y)=l_{S}(S Y) \cap R=Q \cap R$. Also, $l_{S}\left(S / S P^{*}\right) S Y \subseteq S P^{*} Y=0$, so $l_{S}\left(S / S P^{*}\right) \subseteq l_{S}(S Y)=Q$. Consequently,

$$
\begin{aligned}
\mathrm{GK}\left(R / P^{*}\right) & \leq \mathrm{GK}(R / Q \cap R)=\mathrm{GK}(S / Q) \leq \mathrm{GK}\left(S / l_{S}\left(S / S P^{*}\right)\right) \\
& =\mathrm{GK}\left(S / S P^{*}\right)=\mathrm{GK}\left(R / r_{R}\left(S / S P^{*}\right)\right)=\mathrm{GK}\left(R / S P^{*} \cap R\right)=\mathrm{GK}\left(R / P^{*}\right),
\end{aligned}
$$

where we have used [6,5.3 and 5.4], because ${ }_{S}\left(S / S P^{*}\right)_{R}$ is finitely generated on both sides. Thus, $\mathrm{GK}(R / P)=\mathrm{GK}\left(R / P^{*}\right)=\mathrm{GK}(R / Q \cap R)=\mathrm{GK}(S / Q)$. Since $P \supseteq l_{R}(X) \supseteq$ $l_{R}(M)=l_{S}(M) \cap R=Q \cap R, P$ is thus minimal over $Q \cap R$.

We end this section with a simple example that shows that the distinction between left and right lying directly over is necessary.

ExAMPLE 4.9. Let $k$ be a field, let $S=M_{3}(k)$, and let $R$ be the subring of upper triangular $3 \times 3$ matrices over $k$. Note that $R$ and $S$ are noetherian with $\operatorname{GK}(R)=$ $\mathrm{GK}(S)=0$, and both $S_{R}$ and ${ }_{R} S$ are finitely generated. Let

$$
P_{1}=\left[\begin{array}{ccc}
k & k & k \\
0 & k & k \\
0 & 0 & 0
\end{array}\right], \quad P_{2}=\left[\begin{array}{ccc}
k & k & k \\
0 & 0 & k \\
0 & 0 & k
\end{array}\right], \quad P_{3}=\left[\begin{array}{lll}
0 & k & k \\
0 & k & k \\
0 & 0 & k
\end{array}\right]
$$

denote the prime ideals of $R$. Note that 0 is the only prime of $S$, and 0 lies over each $P_{i}$. It is easy to check that

(i) $P_{1} S \cap R=P_{1}, S P_{1} \cap R=R$, so 0 lies right directly, but not left directly over $P_{1}$,

(ii) $S P_{3} \cap R=P_{3}, P_{3} S \cap R=R$, so 0 lies left directly, but not right directly over $P_{3}$,

(iii) $S P_{2} \cap R=P_{2} S \cap R=R$, so 0 lies over $P_{2}$, but neither right nor left directly so. 
5. Finite lying over. It has been pointed out before that in general it is not known whether a prime ideal of $R$ that has LO also has finite LO. In this section, we provide an affirmative answer to this question in some special instances. They involve the relationship of $r(S / R)$ with $P$, or rather the relationship between $\mathrm{GK}(R / r(S / R))$ and $\mathrm{GK}(R / P)$. It is well known that if $R$ and $S$ have a common ideal that is not contained in $P$, then $P$ has LO in the "classical" sense, that is $P=Q \cap R$ for some prime ideal $Q$ of $S$ (see, for example, $[12,2.12 .45])$. Now, $r(S / R)$ is the (unique) largest left ideal of $S$ that is contained in $R$, and it turns out that quite a bit of information can be obtained from the hypothesis that $r(S / R) \nsubseteq P$. Of course, similar results hold when $P$ does not contain $l(S / R)$, the largest right ideal of $S$ that lies in $R$.

PROPOsrrion 5.1. Let $R$ be a subring of a ring $S$, and let $P$ be a prime ideal of $R$ such that $r(S / R) \nsubseteq P$. Then:

(i) $P$ has $L O$;

(ii) if $Q \in \operatorname{spec}(S)$ lies over $P$ and $\operatorname{annspec}(S / Q)_{R}$ is not empty, then annspec $(S / Q)_{R}=\{P\}$, that is, $Q$ lies right directly over $P$, and, furthermore, if in this case $Q$ lies over $P^{\prime} \neq P$, then $r_{R}(S / R) \subseteq P^{\prime}$; LO.

(iii) if $R$ is right (left) noetherian and $S_{R}\left({ }_{R} S\right)$ is finitely generated, then $P$ has finite

Proof. (i) Since $(P S \cap R) r(S / R) \subseteq P$, the hypothesis yields $P S \cap R \subseteq P$, hence $P S \cap R=P$. Set $I=r_{S}(S / P S)$. Then $r(S / R) P \subseteq r(S / P) \subseteq r_{R}(S / P S)=I \cap R \subseteq P S \cap R=P$, and it follows that $P$ is minimal over $I \cap R$. An easy application of Zorn's Lemma shows that there is a prime ideal $Q$ of $S$ such that $P$ is minimal over $Q \cap R$.

(ii) Let $P^{*} \in$ annspec $(S / Q)_{R}, P^{*}=r_{R}(X / Q)$ for some right $R$-submodule $X$ of $S$, $X \subsetneq Q$. Since $X$ may be assumed to be a left ideal of $S$, it follows from $X r(S / R) P^{*} \subseteq$ $X P^{*} \subseteq Q$ that $r(S / R) P^{*} \subseteq Q \cap R \subseteq P$, hence $P^{*} \subseteq P$. As clearly $Q \cap R \subseteq P^{*}$, the minimality of $P$ over $Q \cap R$ forces $P^{*}=P$. Furthermore, if $P^{\prime} \neq P$ is minimal over $Q \cap R$, then $r(S / R) P \subseteq Q \cap R \subseteq P^{\prime}$ implies that $r(S / R) \subseteq P^{\prime}$.

(iii) Assume that $Q \in \operatorname{spec}(S)$ lies over $P \in \operatorname{spec}(R)$. As annspec $(S / Q)_{R} \neq \varnothing$, annspec $(S / Q)_{R}=\{P\}$ by (ii). Again, let $I=r(S / P S)$, and let $P=r_{R}(X / Q)$ with $S \supseteq$ ${ }_{s} X_{R} \subsetneq Q$. Then $X S I \subseteq X P S \subseteq Q$, so $I \subseteq Q$. If $Q$ were not minimal over $I$, say $I \subseteq Q^{\prime} \varsubsetneqq Q$, $Q^{\prime} \in \operatorname{spec}(S)$, then $I \cap R \subseteq Q^{\prime} \cap R \subseteq Q \cap R \subseteq P$. By (i), $P$ is minimal over $I \cap R$, so both $Q^{\prime}$ and $Q$ would lie over $P$. By Corollary 2.4 of [9] this is not possible whenever $R$ is right (left) noetherian and $S_{R}\left({ }_{R} S\right)$ is finitely generated. Thus, any prime ideal of $S$ that lies over $P$ is minimal over $I$. Since $S$ is right (left) noetherian, this establishes (iii).

In [14, Proposition 9] it is shown that if $R \subset S$ is a right and left finite extension of noetherian prime algebras with finite GK-dimension, and if $R_{R}$ is essential in $S_{R}$, then lying over holds for a prime ideal of $P$ of $R$, provided that $\mathrm{GK}(R / P) \geq \mathrm{GK}(S / R)$. Note that if both $S_{R}$ and ${ }_{R} S$ are finitely generated, then $\mathrm{GK}(R / l(S / R))=\mathrm{GK}(S / R)=$ $\mathrm{GK}(R / r(S / R))$. If only $S_{R}$ is assumed to be finitely generated, then one only has that $\mathrm{GK}(R / l(S / R))=\mathrm{GK}_{R}(S / R) \leq \mathrm{GK}(S / R)_{R} \leq \mathrm{GK}(R / r(S / R))$ by $[\mathbf{6}, 5.3$ and 5.4]. Thus, [14, Proposition 9] is a special case of the following.

THEOREM 5.2. Let $R \subset S$ be a right finite extension of noetherian $k$-algebras with finite $G K$-dimension, and let $P$ be a prime ideal of $R$. Then:

(i) if $\mathrm{GK}(R / P) \geq \mathrm{GK}(R / r(S / R))$, then $P$ has finite $L O$; 
(ii) if $\mathrm{GK}(R / P)>\mathrm{GK}_{R}(S / R)$, then there exists a unique prime ideal $Q$ of $S$ that lies over $P$, and $P=Q \cap R$.

Proof. (i) If $r(S / R) \nsubseteq P$, then it is done by Proposition 5.1. If $r(S / R) \subseteq P$, then the hypothesis gives $\mathrm{GK}(R / P)=\mathrm{GK}(R / r(S / R))$. Set $I=l_{S}(S / r(S / R))$, the unique largest two-sided ideal common to $R$ and $S$. By [6,5.5], GK(S/I) $=\mathrm{GK}(R / I)$. Since $R / r(S / R)$ is an $S / I-R / r(S / R)$-bimodule that is faithful and finitely generated on both sides, $\mathrm{GK}(S / I)=\mathrm{GK}(R / r(S / R))$ by $[6,5.3$ and 5.4]. Consequently, $\mathrm{GK}(R / I)=\mathrm{GK}(R / P)$, so $P$ is minimal over $I$. Any ideal of $S$ that contains $I$ and is maximal with respect to the property that its intersection with $R$ is contained in $P$ is then a prime ideal that lies over $P$. Now let $Q$ be any prime ideal of $S$ that lies over $P$. Then $(Q+I) \cap R=(Q \cap R)+I \subseteq$ $P$, hence

$$
\begin{aligned}
\mathrm{GK}(S / Q) & =\mathrm{GK}(R / Q \cap R)=\mathrm{GK}(R / P) \leq \mathrm{GK}(R /(Q+I) \cap R) \\
& =\mathrm{GK}(S / Q+I) \leqslant \mathrm{GK}(S / Q) .
\end{aligned}
$$

Thus, the ideal $Q+I$ of $S$ cannot properly contain $Q$, so $I \subseteq Q$. Finally, as $\mathrm{GK}(S / I)=$ $\mathrm{GK}(R / P)=\mathrm{GK}(S / Q), Q$ must be minimal over $I$. Hence only a finite number of prime ideals of $S$ lie over $P$.

(ii) if $\mathrm{GK}(R / P)>\mathrm{GK}_{R}(S / R)=\mathrm{GK}(R / l(S / R))$, then $l(S / R) \nsubseteq P$, so $P$ has finite LO by the left-handed version of Proposition 5.1. Let $Q$ be a prime of $S$ that lies over $P$. We proceed to show that $Q \cap R=P$ and that $Q$ is unique. Let $P^{*} \in \operatorname{annspec}(S / Q)_{R}$, so $Q$ lies over $P^{*}$ as well, by Proposition 4.1(i). Consequently, $\mathrm{GK}(R / P)=\mathrm{GK}(S / Q)=$ $\mathrm{GK}\left(R / P^{*}\right)$. Now, if $P^{*}=r_{R}(X / Q), Q \subsetneq s X_{R} \subseteq S$, then $P^{*} S \cap R=P^{*}$ and $X l(S / R) P^{*} S \subseteq$ $Q$. Let $X l(S / R) \subseteq Q$. Then $l(S / R) \subseteq P^{*}$, contradicting $\mathrm{GK}\left(R / P^{*}\right)=\mathrm{GK}(R / P)>$ $\mathrm{GK}(R / l(S / R))$. Thus $P^{*} S \subseteq Q$, whence $P^{*}=P^{*} S \cap R \subseteq Q \cap R \subseteq P$, and $P^{*}=Q \cap R=P$ follows. Finally, if $Q^{\prime} \in \operatorname{spec}(S)$ lies over $P$, then $l(S / R) Q^{\prime} \subseteq l(S / R) \cap Q^{\prime} \subseteq Q^{\prime} \cap R \subseteq$ $P=Q \cap R \subseteq Q$. Since $l(S / R) \subseteq Q$ would imply that $l(S / R) \subseteq P$, it follows that $Q^{\prime} \subseteq Q$. Since distinct prime ideals of $S$ that lie over a given prime of $R$ are incomparable by [9, Corollary 2.4], $Q$ is thus the only prime of $S$ lying over $P$.

If $\mathrm{GK}(R / P)<\mathrm{GK}(R / r(S / R)$, then $P$ need not have LO, as is demonstrated by Example 3.1, where $R / P \simeq k$, so $\mathrm{GK}(R / P)=0$, and $r(S / R)=0$, so that $\mathrm{GK}(R / r(S / R))=$ 2. However, if $\mathrm{GK}(R / P)$ is not too small, one still has some information.

PROPOSITION 5.3. Let $R \subseteq S$ be a right finite extension of noetherian $k$-algebras with finite $G K$-dimension, and let $P \in \operatorname{spec}(R)$. Then:

(i) if $P$ is minimal over $r(S / R)$, then $P$ has $L O$;

(ii) if $\mathrm{GK}(R / P)=\mathrm{GK}(R / N)$, where $N / r(S / R)$ denotes the prime radical of $R / r(S / R)$, then $P$ has finite $L O$.

Proof. (i) Let $P$ be minimal over $r(S / R)=l_{R}(R / r(S / R))$. Since $R / r(S / R)$ is a finitely generated left $R$-submodule of the left $S$-module $S / r(S / R), P \in \mathrm{St}_{R}(S / r(S / R))$ whence the claim by Theorem 4.8 .

(ii) If $P \nsupseteq r(S / R)$, then we are done by Proposition 5.1. If $P \supseteq r(S / R)$, then the hypothesis implies that $P$ is minimal over $r(S / R)$, so $P$ has LO by (i). Assume there exist infinitely many primes $Q_{i}$ of $S$ that lie over $P$. For each $Q_{i}$, pick $P_{i} \in \operatorname{annspec}_{R}\left(S / Q_{i}\right)$, 
$P_{i}=l_{R}\left(X_{i} / Q_{i}\right), Q_{i} \varsubsetneqq{ }_{R}\left(X_{i}\right)_{S} \subseteq S$. By Corollary 4.2 , the $P_{i}$ may be assumed to be distinct. For each $i$ we have that $S P_{i} r(S / R) X_{i} \subseteq Q_{i}$, so either $S P_{i} \subseteq Q_{i}$ or $r(S / R) X_{i} \subseteq Q_{i}$. In the first case, $P_{i}=S P_{i} \cap R \subseteq Q_{i} \cap R \subseteq P$, implying $P_{i}=P$ since $\mathrm{GK}\left(R / P_{i}\right)=\mathrm{GK}\left(S / Q_{i}\right)=$ $\mathrm{GK}(R / P)$. As this can happen only for one index $i, r(S / R) X_{i} \subseteq Q_{i}$ and hence $r(S / R) \subseteq P_{i}$ for infinitely many $P_{i}$. Since $\mathrm{GK}\left(R / P_{i}\right)=\mathrm{GK}(R / P)=\mathrm{GK}(R / N), R / r(S / R)$ would thus have infinitely many minimal primes, contradicting the fact that $R$ is noetherian.

Note that if GK-dimension is exact for right $R$-modules, then no separate proof of 5.3(ii) is needed, since then $\mathrm{GK}(R / P)=\mathrm{GK}(R / N)=\mathrm{GK}(R / r(S / R))$ whence the result by $5.2(\mathrm{i})$.

\section{REFERENCES}

1. K. R. Goodearl and E. S. Letzter, Prime ideals in skew and q-skew polynomial rings, Mem. Amer. Math. Soc., No 521 (1994).

2. K. R. Goodearl and R. B. Warfield, Jr., An introduction to noncommutative Noetherian rings, London Mathematical Society Student Texts 16 (Cambridge University Press, 1989).

3. T. J. Hodges and J. Osterlurg, A rank two indecomposable projective module over a Noetherian domain of Krull dimension one, Bull. London Math. Soc. 19 (1987), 139-144.

4. G. Krause, Additive rank functions in Noetherian rings, J. Algebra 130 (1990), 451-461.

5. G. Krause, Prime factor series of modules over a Noetherian algebra, Abelian groups and noncommutative rings, Contemp. Math. 130 (Amer. Math. Soc., 1992), 215-229.

6. G. R. Krause and T. H. Lenagan, Growth of algebras and Gelfand-Kirillov dimension, Research Notes in Mathematics, 116 (Pitman, 1985).

7. T. H. Lenagan and R. B. Warfield, Jr., Affiliated series and extensions of modules, $J$. Algebra 142 (1991), 164-187.

8. E. S. Letzter, Primitive ideals in finite extensions of Noetherian rings, J. London Math. Soc. (2) 39 (1989), 427-435. 412-438.

9. E. S. Letzter, Prime ideals in finite extensions of Noetherian rings, J. Algebra 135 (1990),

10. E. S. Letzter, Finite correspondence of spectra in Noetherian ring extensions, Proc. Amer. Math. Soc. 116 (1992), 645-652.

11. M. Lorenz, $K_{0}$ of skew group rings and simple Noetherian rings without idempotents, $J$. London Math. Soc. (2) 32 (1985), 41-50.

12. L. H. Rowen, Ring theory, Vol. 1 (Academic Press, 1988).

13. L. W. Small, J. T. Stafford and R. B. Warfield, Jr., Affine algebras of Gelfand-Kirillov dimension one are PI, Math. Proc. Cambridge Philos. Soc. 97 (1985), 407-414.

14. L. W. Small and R. B. Warfield, Jr., Finite extensions of rings II, to appear.

15. R. B. Warfield, Jr., Noetherian rings with trace conditions, Trans. Amer. Math. Soc. 331 (1992), 449-463.

Department of Mathematics and Astronomy

THE UNIVERSITY OF MANITOBA

WINNIPEG

MANITOBA R3T 2N2

Canada 